HEFAT2012

$9^{\text {th }}$ International Conference on Heat Transfer, Fluid Mechanics and Thermodynamics

16 - 18 July 2012

Malta

\title{
MULTI EFFECT PLANTS AND IONIC LIQUIDS FOR IMPROVED ABSORPTION CHILLERS
}

\author{
Weith T.*, Preißinger M., Pöllinger S. and Brüggemann D. \\ *Author for correspondence \\ Zentrum für Energietechnik, \\ Universität Bayreuth, \\ Bayreuth, \\ Germany, \\ E-mail: LTTT@uni-bayreuth.de
}

\section{ABSTRACT}

State of the art absorption chillers using conventional working pairs still suffer from problems like crystallization, corrosiveness and a relatively low efficiency. To improve this technology, different working pairs as well as plant designs are investigated using the simulation tool AspenPlus. The simulation is validated by comparing the results of single effect absorption chillers using the current commercially applied working pairs water/lithium bromide and ammonia/water with literature data. To increase the efficiency, double effect absorption chillers are implemented and analyzed. The performance of two kinds of double effect cycles, series and parallel, is compared using the working pair water/lithium bromide. In addition, ionic liquids (ILs) are investigated as a sorbent in order to improve the technology. So far, ILs have not been implemented in AspenPlus yet. Therefore, a guideline for the implementation of ILs in AspenPlus is outlined and the accordant phase equilibria results are validated with literature data. Simulations of single effect cycles using the ILs 1,3-dimethylimidazolium dimethylphosphate ([MMIM][DMP]) and 1-ethyl-3-methylimidazolium dimethylphosphate ([EMIM][DMP]) in combination with water as a refrigerant are performed and the results are compared to conventional working pairs.

\section{INTRODUCTION}

Refrigeration, cooling and air conditioning have always been important issues. Especially the rapid growth of the world population and the increasing conformation of the standard of living of developing countries to industrial nations have led to an enormous increase of cooling demand. This development will additionally be intensified by future climate change.

\section{NOMENCLATURE}

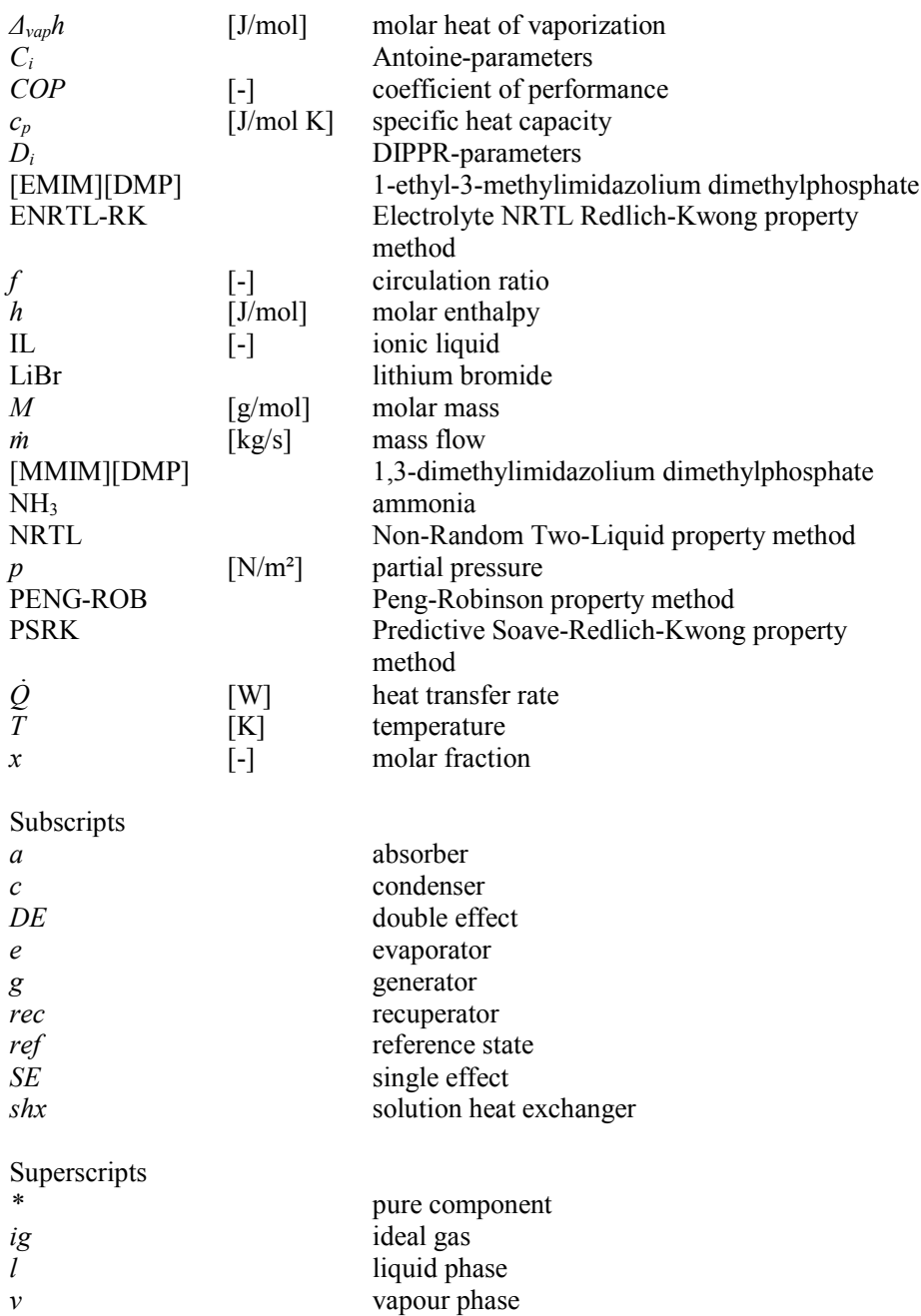


In recent years, conventional compression refrigeration machines have covered the predominant part of cooling need. As it requires a high amount of electrical energy to power these systems, lower energy solutions have to be considered for the future. In this context, absorption chillers represent a suitable alternative to compression devices. These chillers are mainly powered by external heat, whereas the amount of additional electric energy is almost negligible. Therefore, especially in industrial cooling applications where waste heat can serve as heat source, the application of this technology can reduce electric power consumption [1].

Beside this advantage, absorption chillers anyhow suffer from several problems. On the one hand, conventional plant designs generally offer low efficiencies and are limited by the heat source temperature, on the other hand, the state of the art working pairs water/lithium bromide and ammonia/water entail risks like crystallization and corrosion [2]. To overcome these issues, the present work investigates advanced plant designs as well as new working pairs using the simulation tool AspenPlus [3].

In a first step, the accuracy of our simulation is validated by comparing the results of single effect plants using the conventional working pairs water/lithium bromide and ammonia/water with literature data. Later on, the advantages of double effect plants are revealed through the comparative analysis of series and parallel double effect cycles and conventional single effect systems. Finally, as a substitution of conventional working pairs, the ILs 1,3-dimethylimidazolium dimethylphosphate ([MMIM][DMP]) and 1-ethyl-3methylimidazolium dimethylphosphate ([EMIM][DMP]) in combination with water as a refrigerant are used in a single effect absorption system. ILs are chosen as sorbent, due to their generally low vapour pressures and their ability of absorbing huge amounts of refrigerant. Furthermore, corrosion and crystallization problems, as typical for conventional working pairs, can be overcome. ILs have not been implemented in AspenPlus yet. Therefore, the present work outlines a guideline for the implementation of ILs in this simulation tool and validates the accordant phase equilibria results with literature data. Finally, the performance of ILs in single effect absorption chillers is compared with conventional working pairs.

\section{MODELS, METHODS AND BOUNDARY CONDITIONS Models of the Investigated Plant Designs}

The AspenPlus flow sheet of the investigated conventional single effect absorption chiller is represented in Figure 1. The cycle model is set up according to Somers et al. [4] and is realized as a closed loop. In analogy to compression refrigeration systems, the absorption chiller consists of a condenser (COND), an expansion valve (EXPV), an evaporator (EVAP) and a compression unit. In contrast to compression refrigeration chillers, the compression is realized through a socalled thermal compressor through the following steps: In the absorber (ABS), the refrigerant is absorbed by the sorbent. Then, the pump (PMP) raises the solution to a higher pressure level. Before entering the generator unit (GEN) the solution passes the solution heat exchanger ( $\mathrm{SHX}$ ), to reuse a part of the heat introduced in the generator. In GEN the sorbent desorbs the refrigerant again, which then leaves the generator at high pressure.

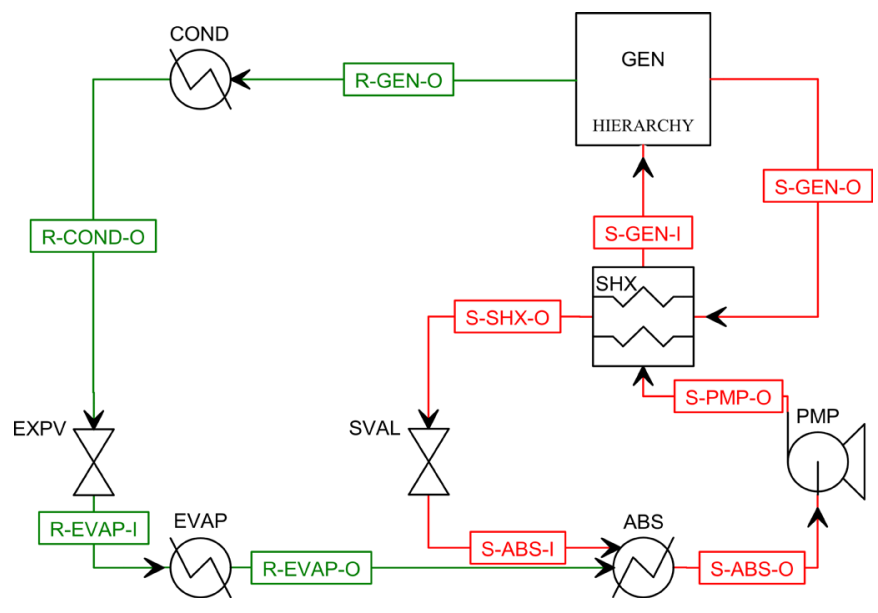

Figure 1 AspenPlus flow sheet of the single effect absorption chiller

Figure 2 shows the generator unit, modelled through a combination of different single components that account for the thermodynamic processes. It should be noted that this set-up does not correspond to a real plant assembly. To fulfil the precondition that the temperature of the refrigerant leaving the generator unit is equal to the boiling temperature of the incoming solution, the first heater (GEH1) heats the solution up to its boiling temperature and transfers this value to the heat exchanger (GENX). There, the vapour transfers as much heat to the solution as required to reach its boiling temperature. The flash unit (GENF) separates the evaporated refrigerant and the residual solution and the superheated vapour leaves the generator through the heat exchanger.

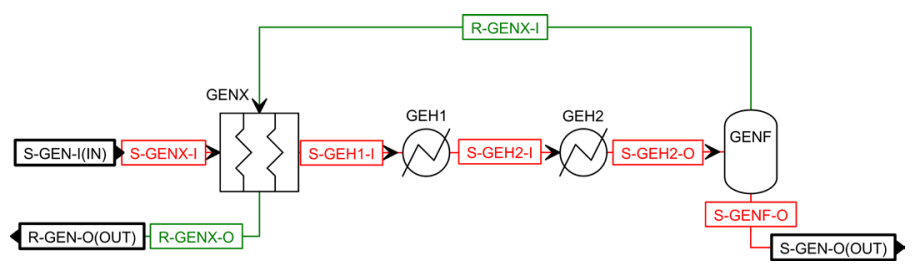

Figure 2 AspenPlus flow sheet of the generator hierarchy

The performance of absorption chillers is expressed as coefficient of performance $(C O P)$. Neglecting the small power consumption of the pump, it can be calculated in terms of the ratio of evaporator and generator duty:

$$
C O P=\frac{\dot{Q}_{E V A P}}{\dot{Q}_{G E N}}=\frac{\dot{Q}_{E V A P}}{\dot{Q}_{G E H 1}+\dot{Q}_{G E H 2}}
$$

The second investigated plant design is the single effect system with an additional rectification as represented in Figure 3. This configuration has to be chosen in case of ammonia/water as a working pair. As the vapour pressure of water is not negligible compared to that of ammonia, the vapour generated in the desorber still contains a small fraction of water. Hence, for reducing this amount and raising the $C O P$, the rectification column has to be added. 


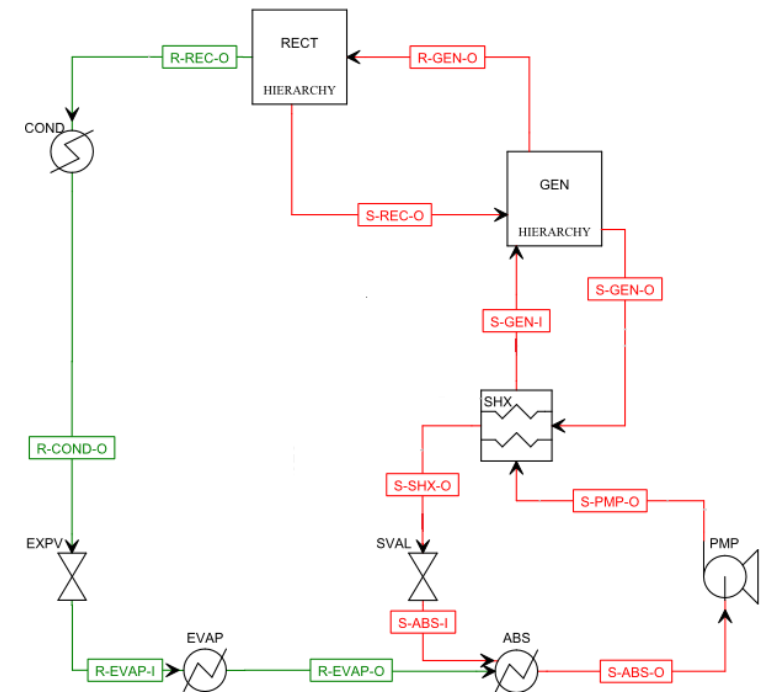

Figure 3 AspenPlus flow sheet of the single effect absorption chiller with rectification

Due to the rectification, the generator possesses an additional inflow stream. Therefore, its model has to be adjusted. The flow sheet in Figure 4 shows a supplemental mixer (MIX) and flash unit (GEF2).

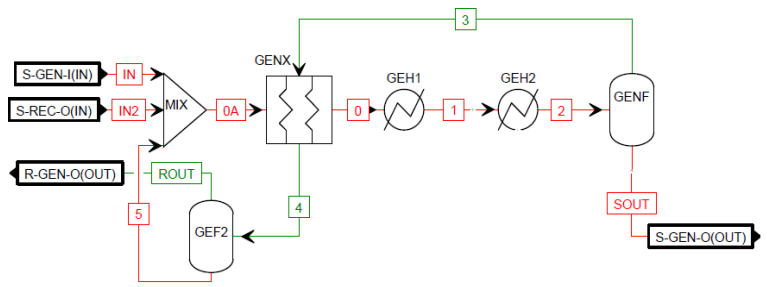

Figure 4 AspenPlus flow sheet of generator hierarchy in case of an additional rectification

In the mixer, the solution stream of the solution heat exchanger, the reflow from the rectification and the liquid phase from GEF2 converge. Vapour stream 3 still contains small fractions of water. A part of that liquid can already be condensed out in GENX. This condensate is then separated in GEF2 and is fed back to GENX by MIX.

The modelling of the required rectification unit in AspenPlus is illustrated in Figure 5. It consists of two flash blocks, a mixer, as well as a heater and a cooler.

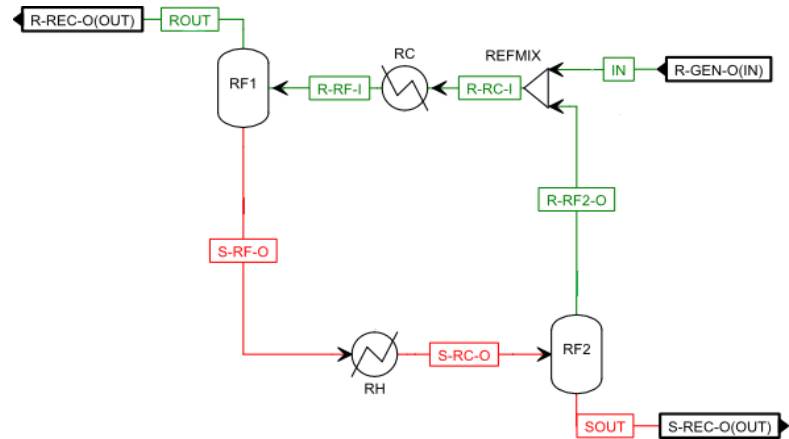

Figure 5 AspenPlus flow sheet of the rectification hierarchy
The condensate coming from the flash block RF1 is partly evaporated by the heater RH. In the flash device RF2 the vapour fraction is then separated from the residual liquid phase, which flows back to the generator. In REFMIX the vapour is mixed up with the vapour coming from the generator. Thereafter, it is partly condensed in RC. According to vapourliquid equilibrium, the content of sorbent in the liquid phase exceeds that in the vapour phase. The purity of the refrigeration vapour is set by defining the temperature of RC. Finally, the condensate is separated in RF1 and reflows through the rectification cycle, whereas the refrigerant-rich vapour leaves the rectification.

As the described rectification requires an additional heat input in $\mathrm{RH}$, the $\mathrm{COP}$ for the $\mathrm{NH}_{3}$ /water absorption chiller is redefined as follows:

$$
C O P=\frac{\dot{Q}_{E V A P}}{\dot{Q}_{G E N}}=\frac{\dot{Q}_{E V A P}}{\dot{Q}_{G E H 1}+\dot{Q}_{G E H 2}+\dot{Q}_{R H}}
$$

Next to the described single effect plant configurations, double effect designs using water/ $\mathrm{LiBr}$ as a working pair are investigated.

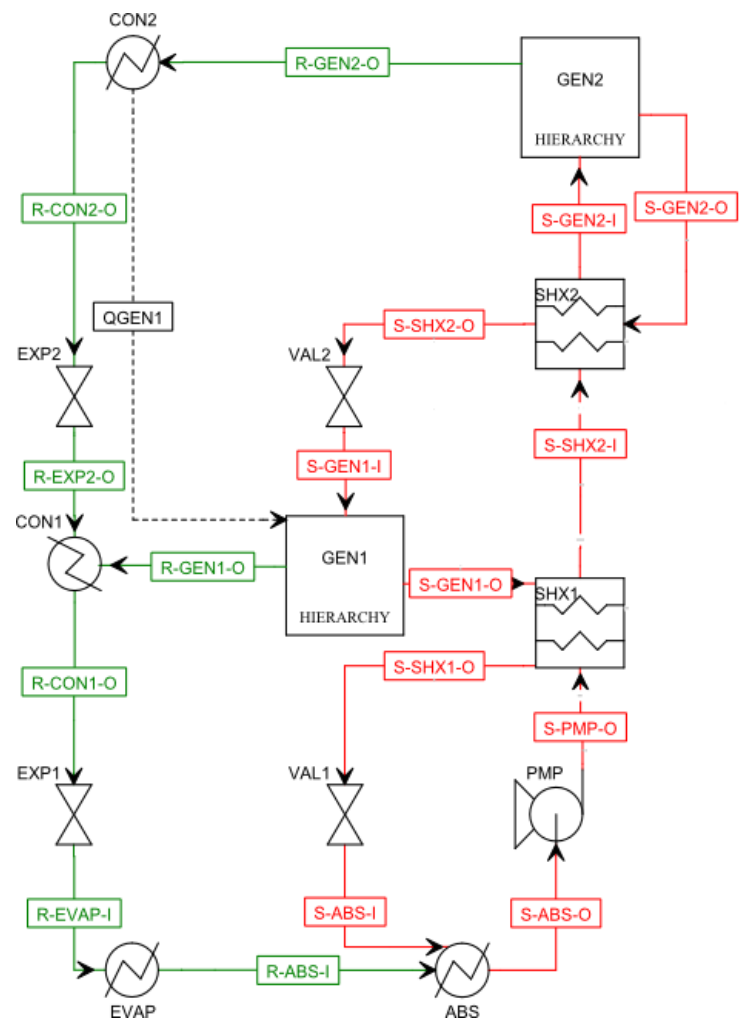

Figure 6 AspenPlus flow sheet of the series double effect absorption chiller

As in single effect plants, considering water $/ \mathrm{LiBr}$, no additional rectification unit is needed. Double effect plants can be regarded as a combination of two single effect systems and the resulting $C O P$ can be estimated in terms of the single effect COP [1]:

$$
C O P_{D E}=C O P_{S E}+C O P_{S E}{ }^{2}
$$


The double effect cycle technology distinguishes several variants. These mainly vary in the kind of connection of the two solution cycles. In this study, series and parallel configurations are investigated.

The applied model of the series double effect plant is represented in Figure 6. In this plant design the second generator GEN2 corresponds to the single effect generator shown in Figure 2. In the first generator (GEN1) the heat of condensation of condenser CON2 is used to desorb even more refrigerant. Its AspenPlus realization is shown in Figure 7.

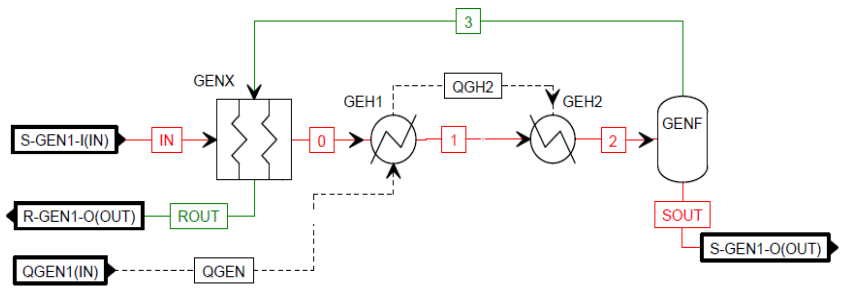

Figure 7 AspenPlus flow sheet of generator hierarchy GEN1 of the series double effect configuration

Figure 8 presents the double effect plant in parallel configuration. GEN2 again equals that of the single effect plant, while GEN1 is modified by an additional splitter. Its hierarchy is visible in Figure 9.

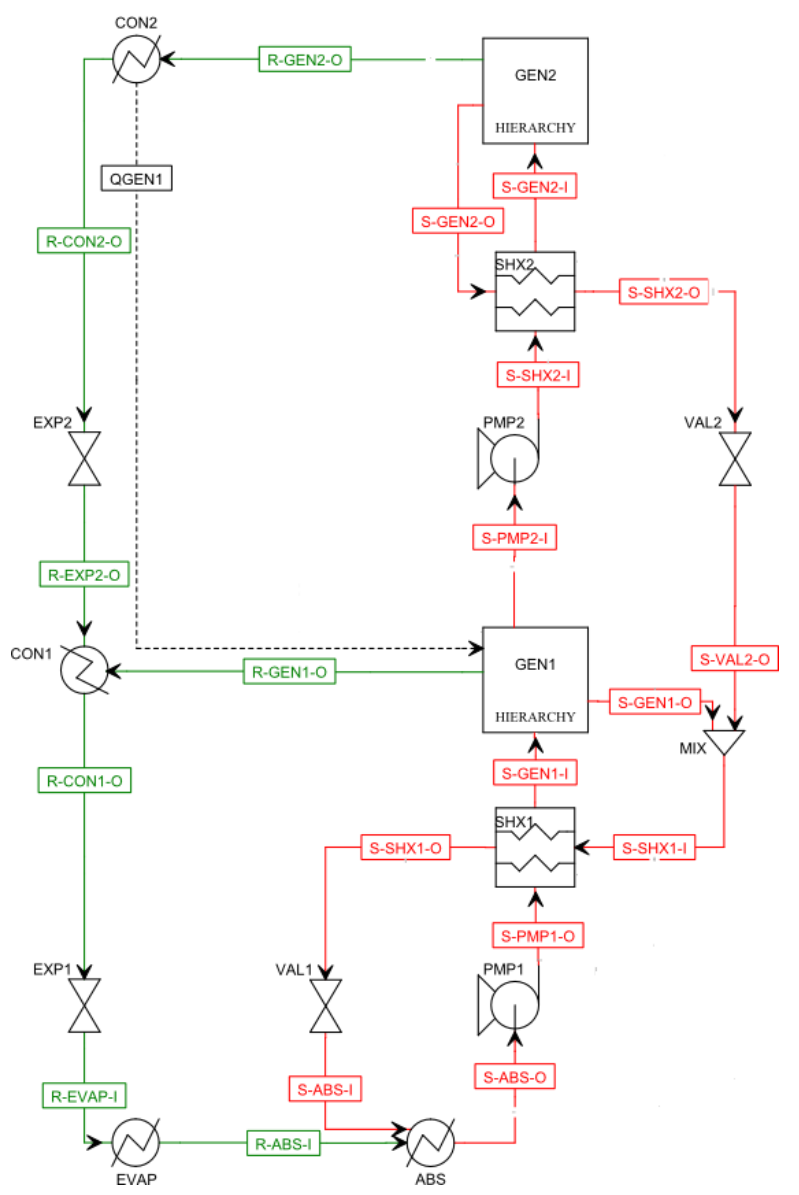

Figure 8 AspenPlus flow sheet of parallel double effect absorption chiller

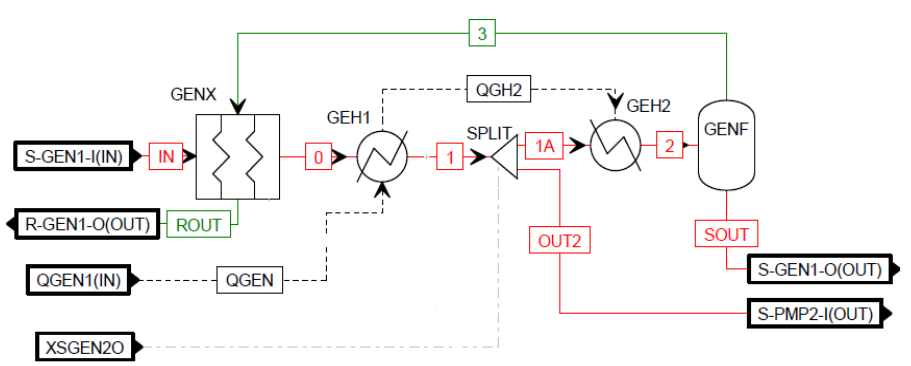

Figure 9 AspenPlus flow sheet of generator hierarchy GEN1 of the parallel double effect configuration

The calculation of the $C O P$ of the double effect cycles follows equation (1). The generator duties in the denominator of this equation then correspond to the second generator GEN2.

\section{Boundary Conditions}

In the present study, the refrigerant is fully liquefied $(x=0)$ in the condenser at a condensation temperature of $40{ }^{\circ} \mathrm{C}$ and fully evaporated $(x=1)$ in case of conventional working pairs, respectively evaporated to a vapour fraction of $x=0.99999$ in case of ILs, in the evaporator at an evaporation temperature of $10{ }^{\circ} \mathrm{C}$. Setting the evaporator temperature to a fixed value corresponds to the aim of achieving a desired cooling temperature. The absorption temperature is set to $35^{\circ} \mathrm{C}$ and in the solution heat exchanger, a pinch point difference of $10^{\circ} \mathrm{C}$ is assumed.

Regarding the double effect cycles, the boundary conditions of condenser and generator are related to CON2 and GEN2 respectively.

\section{Property Methods}

For water/LiBr the property model ENRTL-RK is used, as it is designed for describing electrolytes. This method is based on the Asymmetric Electrolyte NRTL property model. It utilizes the Redlich-Kwong equation of state for vapour phase properties and the asymmetric reference state for ionic species (infinite dilution in aqueous solution) [5].

In case of the working pair ammonia/water, the property model PENG-ROB is applied. For the purpose of comparison, simulations are carried out by using the NRTL- and PSRKmodel as well.

Regarding ILs, the standard NRTL-model is assumed, which applies the NRTL activity coefficient model for liquid phase and the ideal gas equation of state for vapour phase [5].

\section{Guideline for the Implementation of ILs in AspenPlus}

As mentioned at the beginning, ILs are not available in the AspenPlus databanks. Therefore, a methodology to implement these fluids had to be developed. The procedure proposed in this study follows Seiler et al. [6]. Figure 10 shows the major steps together with the required input data. 


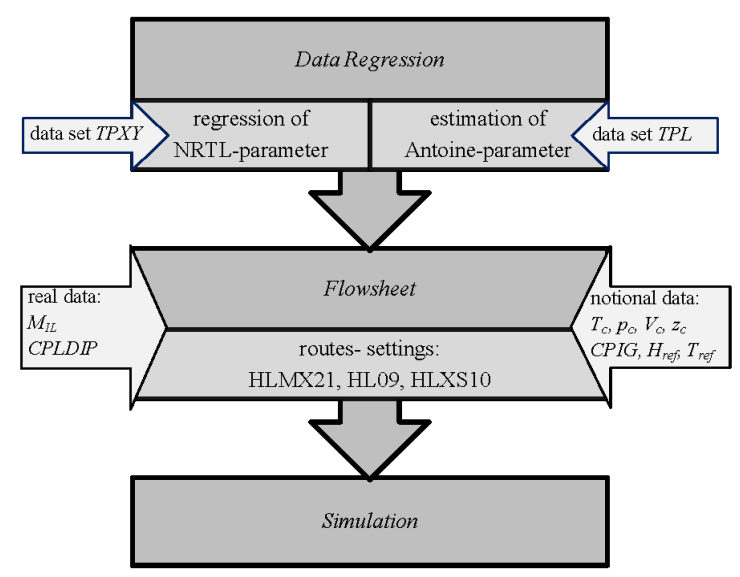

Figure 10 Guideline for the implementation of ILs in AspenPlus

As the NRTL-model is used in our simulation, the first step is to generate the NRTL-parameters through data regression. For this purpose, several fluid properties are required. These include vapour liquid equilibrium (VLE) data and a data set for the saturation pressure. The VLE data points in form of TPXY values originate from $\mathrm{He}$ et al. [7] for water/[MMIM][DMP] and from Ren et al. [8] in case of water/[EMIM][DMP], respectively. The saturation pressure as a function of temperature is defined by the extended Antoine equation:

$$
\ln p_{i}^{*, l}=C_{1 i}+\frac{C_{2 i}}{T+C_{3 i}}+C_{4 i} T+C_{5 i} \ln T+C_{6 i} T^{C_{7 i}}
$$

The coefficients in this equation are estimated under the use of a notional data set $(T P L)$. Due to the extremely low vapour pressure of ILs these fictive values $\left(0^{\circ} \mathrm{C} / 1 \cdot 10^{-9}\right.$ bar and $100^{\circ} \mathrm{C} / 2 \cdot 10^{-9}$ bar) do not influence the calculations significantly.

The second step is relevant to determine enthalpies correctly. The standard NRTL model uses the ideal gas enthalpy for calculating liquid phase properties. For ILs information about the critical point and the ideal gas heat capacity is not available and, therefore, it is not possible to calculate vapour phase. As a consequence, the routes defining the calculation methods in AspenPlus have to be changed so that all relevant properties are calculated from liquid phase data. Thus, the route for calculating the liquid molar enthalpy of a mixture HLMX is set to HLMX21 so that it is expressed in terms of the liquid enthalpies of the pure components and their molar fractions:

$$
h^{l}(T)=\sum_{i} x_{i} h_{i}^{*, l}(T)
$$

For the calculation of liquid enthalpies of pure components the liquid heat capacity is integrated from a reference temperature to the actual temperature and a reference enthalpy is added by setting the route HL to HL09:

$$
h_{i}^{*, l}(T)=h_{i}^{*, l}\left(T_{r e f}\right)+\int_{T_{r e f}}^{T} c_{p, i}^{*, l}(T) M d T
$$

The reference enthalpy is determined by the sum of ideal gas enthalpy and the departure of the vapour phase from the ideal behaviour minus the heat of vaporization:

$$
h_{i}^{*, l}\left(T_{r e f}\right)=h_{i}^{*, i g}+\left(h_{i}^{*, v}-h_{i}^{*, i g}\right)-\Delta_{\text {vap }} h_{i}^{*, l}
$$

As the deviation from the ideal behaviour is calculated through the ideal gas model it vanishes in equation (7). The reference enthalpy is a constant value as it is defined at fixed reference temperature. Therefore, calculation errors just affect absolute enthalpy values. As in these processes only enthalpy differences are important for energy balances and the $C O P$, the calculation of the reference enthalpy can be based on pseudo data and parameters for the ideal gas heat capacity $(C P I G)$ can be notional. The Watson model is used for calculating the heat of vaporization. For the reference enthalpy $\left(h_{r e f}\right)$ and temperature $\left(T_{r e f}\right)$ required in this method notional values are used, too. Finally, for calculating the liquid heat capacity of the pure component required in equation (6), the DIPPR-model is used:

$$
c_{p, i}^{*, l}(T)=D_{1 i}+D_{2 i} T+D_{3 i} T^{2}+D_{4 i} T^{3}+D_{5 i} T^{4}
$$

The coefficients in this polynomial (CPLDIP) are gained by the regression of experimental data from He and Ren using MATLAB [9]. The last route that has to be set is that of the excess enthalpy HLXS. The default setting assumes a zero excess enthalpy. The only way to determine the excess enthalpy without additional fluid properties consists in HLXS10, which utilizes the NRTL-model.

Next to the described ones, further properties necessary for the simulation have to be implemented. These include the molar mass that can be found in data sheets or literature and critical parameters like $T_{\text {crit }}, p_{\text {crit }}, V_{\text {crit }}$ and $z_{\text {crit }}$, for which notional values have to be assumed.

In the end, the AspenPlus sheet containing all the necessary data and calculation methods is copied in the AspenPlus plant design project. Henceforth, simulations with ILs as sorbents in absorption chillers can be performed.

\section{VALIDATION}

\section{Validation of the Single Effect Cycles}

To validate our simulations, comparisons between our results and Herold et al. [10] have been drawn. Contrary to the boundary conditions described above, conditions were set equal to the reference. The results for water $/ \mathrm{LiBr}$ are presented in Table 1. The generator duty is set fixed. Hence, the values solely vary in the range of the tolerance lodged in AspenPlus. The duties of condenser and evaporator deviate by nearly $4 \%$. This results from an increased mass flow in the refrigerant branch rooted in the difference in calculating phase equilibria. The deviation in the mass flow is the reason for the decreased circulation ratio, too. Due to the increased evaporator duty and the nearly constant generator duty, the $C O P$ reaches higher values in the AspenPlus simulations. 
Table 1 Comparison of our results for water/LiBr with Herold et al. [10]

\begin{tabular}{lrrr}
\hline & Herold et al. & this work & $\varepsilon_{\text {rel }}[\%]$ \\
\hline$\dot{Q}_{a}$ in kW & 14.297 & 14.274 & -0.16 \\
$\dot{Q}_{c}$ in kW & 11.427 & 11.858 & 3.77 \\
$\dot{Q}_{g}$ in $\mathrm{kW}$ & 14.952 & 14.948 & -0.02 \\
$\dot{Q}_{e}$ in $\mathrm{kW}$ & 10.772 & 11.183 & 3.82 \\
$\dot{Q}_{s h x}$ in $\mathrm{kW}$ & 3.09 & 3.17 & 2.46 \\
$C O P$ & 0.72 & 0.748 & 3.90 \\
$f$ & 10.837 & 10.423 & -3.82 \\
\hline
\end{tabular}

Regarding ammonia/water as a working pair, the purity of ammonia is set as a fixed condition. Consequently, the mass flow rates in the refrigerant branch are consistent. Table 2 shows the deviations of our simulations using PENG-ROB as a property method in comparison to the reference data.

Despite the almost equal refrigerant mass flow rate, the values for the condenser duty differ by around $4.9 \%$, whereas the evaporator duty does not deviate significantly. Using other property methods like the NRTL or PSRK model, different deviations occur. Therefore, as all of them are based on the same plant model, differences in phase equilibria calculations are the reason for these deviations, too. This is emphasized by regarding the duties of absorber and generator. Especially the duty of the rectification shows with $26.5 \%$ a high deviation. Using other property methods like for example the NRTLmodel it differs by merely $1.14 \%$. Therefore, it is obvious that again differences in the calculation of phase equlibria cause this effect. Notably high differences also arise at the solution heat exchanger with nearly $11 \%$. The absorber temperature is nearly equal to the literature value, but the generator temperature is much higher. Moreover, the heat exchanger efficiency is fixed. Hence, the temperature difference of the refrigerant-poor solution and therewith the transferred duty is increased. It should be noted that in this case the $C O P$ is calculated according to equation (1), as Herold et al. did so. Hence, the additional rectification is not considered.

Table 2 Comparison of our results for $\mathrm{NH}_{3} /$ water with Herold et al. [10]

\begin{tabular}{lrrr}
\hline & Herold et al. & this work & $\varepsilon_{\text {rel }}[\%]$ \\
\hline$\dot{Q}_{a}$ in $\mathrm{kW}$ & 216 & 200.37 & -7.24 \\
$\dot{Q}_{c}$ in $\mathrm{kW}$ & 151 & 158.35 & 4.87 \\
$\dot{Q}_{g}$ in $\mathrm{kW}$ & 268 & 242.99 & -9.33 \\
$\dot{Q}_{e}$ in $\mathrm{kW}$ & 147 & 147.80 & 0.54 \\
$\dot{Q}_{\text {shx }}$ in $\mathrm{kW}$ & 346 & 383.36 & 10.80 \\
$\dot{Q}_{\text {rec }}$ in $\mathrm{kW}$ & 51 & 37.49 & -26.50 \\
$C O P$ & 0.549 & 0.608 & 10.79 \\
$f$ & 7.31 & 7.31 & 0.07 \\
\hline
\end{tabular}

Besides the single effect chillers, double effect configurations have been validated, too. None of the resulting deviations reaches values above $9 \%$.

Summing up it can be concluded that the developed plant models together with the property methods chosen provide comparable results to literature data and are qualified for further investigations.

\section{Validation of the Implementation of ILs}

To review the regression of the NRTL-parameters and, therefore, the correct implementation of ILs in AspenPlus, phase equilibria data of the ternary mixture water, ethanol and $[\mathrm{EMIM}]\left[\mathrm{BF}_{4}\right]$ are compared to literature data provided by Seiler et al. [6]. As a regression method the maximumlikelyhood method is chosen in AspenPlus. Figure 11 shows the results of our simulation together with the experimental and calculated values from Seiler et al. at a temperature of $90{ }^{\circ} \mathrm{C}$ for a molar fraction of the IL of 0.1 and 0.5 respectively.

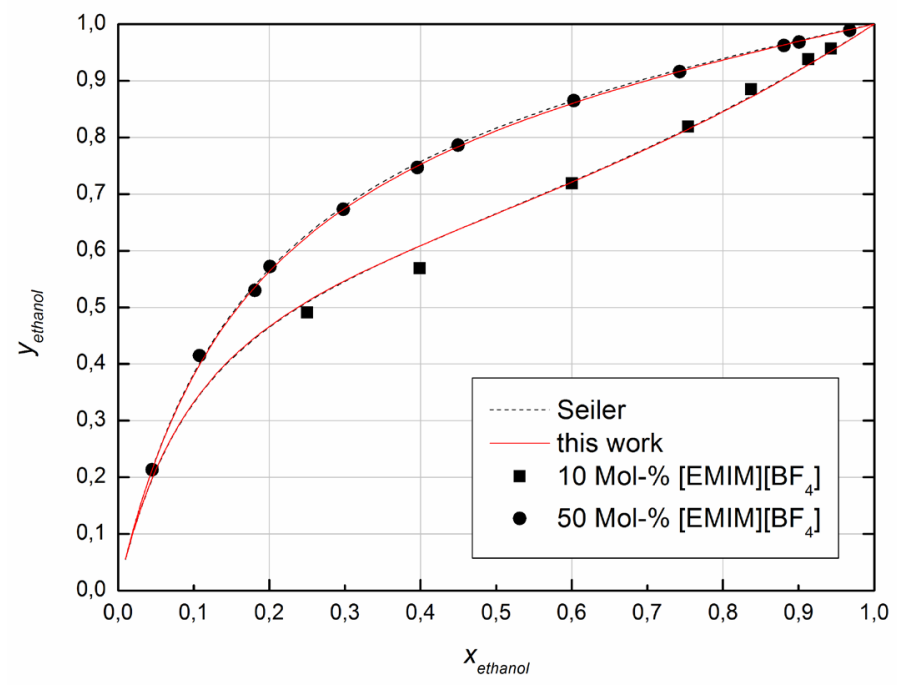

Figure 11 y,x-diagram for the system ethanol-water$[\mathrm{EMIM}]\left[\mathrm{BF}_{4}\right]$

It can be seen that our results generated with AspenPlus correspond well to the results Seiler calculated by the use of NRTL-parameters. Consequently, in both cases the deviation from experimental data is almost equal.

Next to the correct calculation of phase equilibria, the effect of the notional data used during the implementation has been investigated. It could be concluded through a simple simulation of transferring heat to a mixture of water and [EMIM] $\left[\mathrm{BF}_{4}\right]$ in a flash-device that the variation of these values has no significant influence on the results.

\section{RESULTS AND DISCUSSION \\ Comparison of Double Effect Cycles}

Figure 12 presents the results of the single and double effect absorption chillers using water/LiBr as a working pair. Boundary conditions are set as described in the corresponding sub-chapter and the $C O P$ is calculated according to equation (1). 
The diagram shows the variation of the $C O P$ with rising generator temperature. Next to the attained simulation data, theoretical values for the double-effect plant calculated by equation (3) are represented by the hollow symbols.

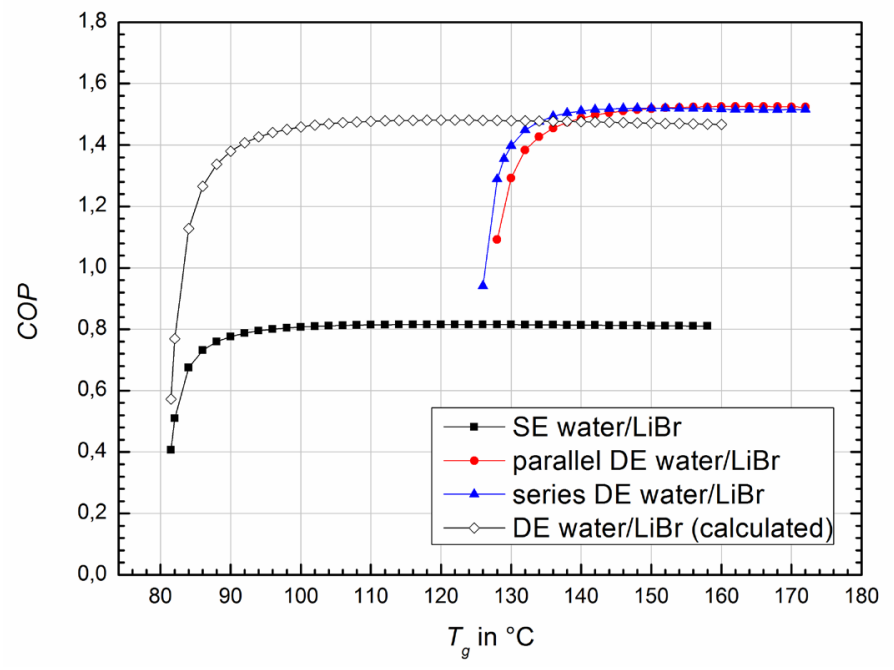

Figure 12 Influence of the generator temperature on the $C O P$ of different plant designs using water/LiBr as working pair

At comparable generator temperatures, a relatively good concordance between these predicted values and the simulation data for the double-effect cycles can be noticed. Therefore, a $C O P$ increase of up to about $88 \%$ can be achieved by using double effect cycles.

Comparing series and parallel configuration, only small differences can be determined. For small generator temperatures, the $C O P$ of the series configuration exceeds the one of the parallel design by maximal $5 \%$ regarding reasonable generator temperatures. This trend changes at a temperature of $152{ }^{\circ} \mathrm{C}$, where for the first time the parallel cycle is superior to the series. The parallel one reaches in this range a COP increase of maximal $0.8 \%$ compared to the series cycle. In analogy to the results reported by Xu and Dai [11] this change is likely to depend on the solution heat exchanger heat-recover effectiveness. As the parallel cycle requires more control complexity [10] and $C O P$-values of both configurations are quite similar, series designs should be favored.

Parallel to that, the figure shows a shift to higher generator temperatures in case of the double effect cycles. This is consistent with literature statements that double effect absorption chillers are employed at high heat source temperatures $[10 ; 12]$.

It has to be mentioned that the above results of water $/ \mathrm{LiBr}$ are quite theoretical, as they do not consider a possible crystallization. According to Herold et al. [10] water/LiBr may crystallize at generator temperatures above $100{ }^{\circ} \mathrm{C}$ under the investigated boundary conditions considering the single-effect chiller. Farshi et al. [2] report a less risk of crystallization for double effect cycles. They also concluded that parallel configuration shows the widest operating range without crystallization. Nevertheless, this risk has to be kept in mind when comparing systems containing water $/ \mathrm{LiBr}$.
Regarding the single-effect ammonia/water absorption chiller, operating temperatures are in the same range as in the investigated double effect systems. However, $C O P$-values are far beyond the ones of the double effect plants as shown in Figure 13.

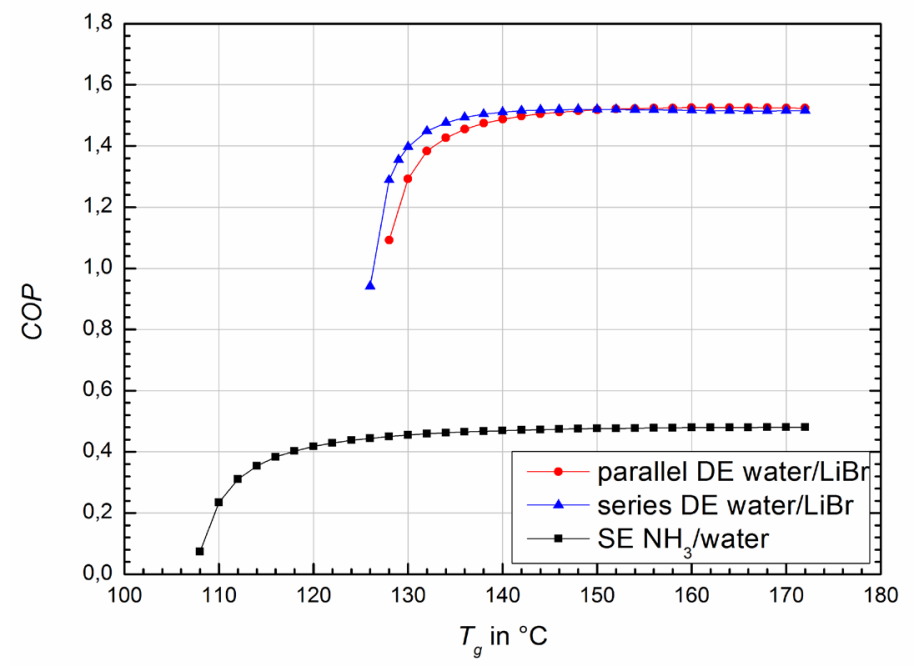

Figure 13 Influence of the generator temperature on the $C O P$ of double effect systems using water $/ \mathrm{LiBr}$ and a single effect plant using ammonia/water

\section{Effect of ILs as Sorbent}

The effect of the generator temperature on the $C O P$ of the single effect plants using the ILs [MMIM][DMP] and [EMIM][DMP] in combination with water as a working pair is represented in Figure 14. For the purpose of comparison, the results of the single effect configurations with conventional working pairs are illustrated, too.

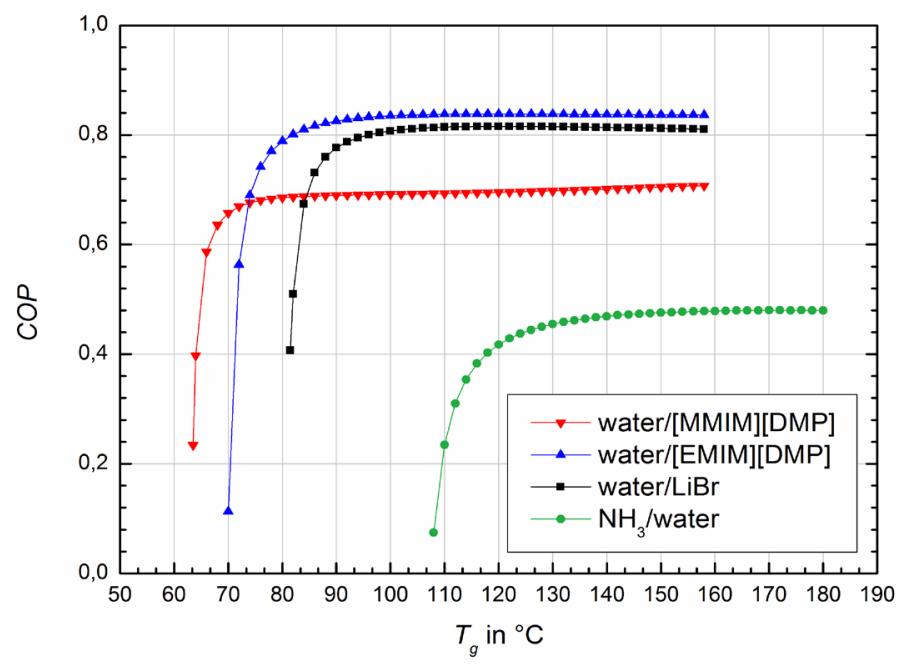

Figure 14 Influence of the generator temperature on the $C O P$ of a single effect absorption chiller using different working pairs 
It can be seen that the working pair ammonia/water again cannot compete with the others, whereas the COPs of water $/ \mathrm{LiBr}$ are comparable to that of the ILs. Water/[EMIM][DMP] seems to be the most promising pair as it reaches the highest $C O P$-values with a maximum of 0.84 . Similar results with regard to ILs have been found by other research groups (Yokoseki and Shiflett [13]). Next to the increased $C O P$, the IL provides a wider operating range. While it can efficiently be used up from a generator temperature of about $75^{\circ} \mathrm{C}$, water/LiBr needs temperatures above $85^{\circ} \mathrm{C}$ and ammonia/water even around $115^{\circ} \mathrm{C}$. Therefore, applying this IL allows the use of minor heat source temperatures. Regarding water/[MMIM][DMP] this range can be enlarged down to a temperature of $65^{\circ} \mathrm{C}$, but the maximal achievable $C O P$ value of around 0.71 is below that of water/[EMIM][DMP] and water/LiBr.

Again, regarding water/LiBr, the risk of crystallization has to be taken into account at generator temperatures above $100{ }^{\circ} \mathrm{C}$.

\section{CONCLUSION}

Simulations of various absorption chillers have been performed considering different plant design as well as working pairs. The comparison of the results of single effect absorption chillers using water/LiBr and ammonia/water with literature data revealed good agreement. Based on the correct implementation of the processes, double effect cycles have been simulated. The results show that these cycles are suitable for high heat source temperatures. Though the $C O P$ reaches almost 1.9 times the $C O P$ of the single effect cycle, a more complex plant design has to be considered. Between series and parallel configuration only small differences can be observed in the COP. Moreover, the superiority of one configuration changes at a certain generator temperature depending on the solution heat exchanger effectiveness.

Regarding ILs as solvent, their correct implementation in AspenPlus could be verified. The results of the single effect absorption chiller using ILs showed significantly higher COPs compared to ammonia/water and similar ones to water $/ \mathrm{LiBr}$. [EMIM][DMP] reaches higher values than water $/ \mathrm{LiBr}$ (up to 0.84), whereas with a maximum $C O P$ of 0.71 [MMIM][DMP] provides minor values. Apart from this, ILs show a broader operation range. In case of [MMIM][DMP] an effective use starts at around $65^{\circ} \mathrm{C}$, whereas water/ $\mathrm{LiBr}$ requires temperatures above $85^{\circ} \mathrm{C}$ and ammonia/water even $115^{\circ} \mathrm{C}$. As the two investigated ILs show different operating ranges as well as $C O P \mathrm{~s}$, it is obvious to find even more promising ILs out of the presently over 1500 species in the future [14].

Summing up it can be concluded that ILs provide with their similar or even higher COPs and their broader operating range a promising alternative to the problematic conventional working pairs in the absorption refrigeration technology. Furthermore, double effect systems have been detected as an instrument to improve efficiency. Therefore, a combination of both aspects seems to represent a promising solution. Simulations of ILs in double effect absorption chillers will be carried out in the future to further improve the absorption refrigeration technology and make it even more competitive compared to conventional compression refrigeration machines. Moreover, experimental measurements of properties of ILs will provide a proper data base to increase the accuracy of the AspenPlus results.

\section{REFERENCES}

[1] Srikhirin P., Aphornratana S., Chungpaibulpatana S., A review of absorption refrigeration technologies, Renewable and Sustainable Energy Reviews 5 (4), 2001, pp. 343-372

[2] Garousi Farshi L., Seyed Mahmoudi S.M., Rosen M.A., Analysis of crystallization risk in double effect absorption refrigeration systems, Applied Thermal Engineering 31 (10), 2011, pp. 1712-1717

[3] Aspen Technology, Incorporation. Aspen One V 7, Process Optimization for Engineering, Manufacturing, and Supply Chain, Aspen Plus V 7.3., 2011

[4] Somers C., Mortazavi A., Hwang Y., Radermacher R., Rodgers P., Al-Hashimi S., Modeling water/lithium bromide absorption chillers in ASPEN Plus, Applied Energy 88 (11), 2011, pp. 4197-4205

[5] Aspen Technology Inc., Aspen Physical Property System. Physical Property Methods. Documentation to AspenPlus V 7.3, Burlington, 2011

[6] Seiler M., Jork C., Schneider T., Arlt W. (Hg.), Ionic liquids and hyperbranched polymers - promising new classes of selective entrainers for extractive distillation, Proceedings of the International Conference on Distillation and Absorption, 2002

[7] He Z., Zhao Z., Zhang X., Feng H., Thermodynamic properties of new heat pump working pairs, 1,3-Dimethylimidazolium dimethylphosphate and water, ethanol and methanol, Fluid Phase Equilibria 298 (1), 2010, pp. 83-91

[8] Ren J., Zhao Z., Zhang X., Vapor pressures, excess enthalpies, and specific heat capacities of the binary working pairs containing the ionic liquid 1-ethyl-3-methylimidazolium dimethylphosphate, The Journal of Chemical Thermodynamics 43 (4), 2011, pp. 576-583

[9] MathWorks, Inc.: MATLAB R2010b, 2010

[10] Herold K.E., Radermacher R., Klein S.A., Absorption chillers and heat pumps, Boca Raton, CRC Press, 1996

[11] Xu G.P., Dai Y.Q., Theoretical analysis and optimization of a double-effect parallel-flow-type absorption chiller, Applied Thermal Engineering 17 (2), 1997, pp. 157-170

[12] Arun M.B, Maiya M.P, Srinivasa Murthy S., Performance comparison of double-effect parallel-flow and series flow waterlithium bromide absorption systems, Applied Thermal Engineering 21 (12), 2001, pp. 1273-1279

[13] Yokozeki A., Shiflett M.B., Water Solubility in Ionic Liquids and Application to Absorption Cycles, Ind. Eng. Chem. Res 49 (19), 2010, pp. 9496-9503

[14] Freemantle M., An Introduction to Ionic Liquids, Cambridge, RSC Pub., 2010 